\title{
Effects of low-frequency ultrasound and microbubbles on angiogenesis-associated proteins in subcutaneous tumors of nude mice
}

\author{
ZHI-YONG SHEN $^{1,2}$, E SHEN $^{1}$, JI-ZHEN ZHANG ${ }^{1}$, WEN-KUN BAI ${ }^{1}$, YU WANG $^{1}$, \\ SHAO-LIN YANG ${ }^{1}$, SHU-LIANG NAN ${ }^{1}$, YAN-DUAN LIN ${ }^{1}$, YI LI ${ }^{1}$ and BING HU ${ }^{1}$ \\ ${ }^{1}$ Department of Ultrasound in Medicine, Shanghai Institute of Ultrasound in Medicine, \\ Shanghai Jiaotong University Affiliated Sixth People's Hospital, Shanghai Jiaotong University \\ School of Medicine, Shanghai 200233; ${ }^{2}$ Department of Radiology, Nantong University \\ Affiliated Nantong Tumor Hospital, Nantong, Jiangsu 226361, P.R. China
}

Received February 26, 2013; Accepted April 22, 2013

DOI: $10.3892 / o r .2013 .2492$

\begin{abstract}
It has been shown that 1 and $3 \mathrm{MHz}$ low-intensity ultrasound was able to affect the fragile and leaky angiogenic blood vessels in a tumor. However, the biological effects of $21 \mathrm{kHz}$ low-intensity ultrasound on tumors remain unclear. The aim of the present study was to explore the effects of $21 \mathrm{kHz}$ ultrasound with microbubbles on the regulation of vascular endothelial growth factor (VEGF), cyclooxygenase-2 (COX-2) and apoptosis in subcutaneous prostate tumors in nude mice. The study included three parts, each with 20 tumor-bearing nude mice. Twenty nude mice were divided into four groups: control (sham treatment), microbubble ultrasound contrast agent (UCA), low-frequency ultrasound (US) and US+UCA groups. The UCA used was a microbubble contrast agent (SonoVue). The parameter of ultrasound: $21 \mathrm{kHz}$, an intensity of $26 \mathrm{~mW} / \mathrm{cm}^{2}, 40 \%$ duty cycle (on $2 \mathrm{sec}$, off $3 \mathrm{sec}$ ), $3 \mathrm{~min}$, once every other day for 2 weeks. In the first study, all subcutaneous tumors were examined by contrast-enhanced ultrasonography (CEUS) at the initiation and completion of the experiments. Peak intensity (PI), time to peak intensity (TTP) and area under the curve (AUC) on the time intensity curve (TIC) were analyzed. In the second study, the intensity of VEGF and COX-2 protein expression in the vascular endothelium and cytoplasm was evaluated using immunohistochemistry and laser confocal microscopy. In the third study, terminal deoxynucleotidyl transferase (TdT)-mediated dUTP nick end labeling (TUNEL) assay was used for the evaluation of cell apoptosis in tumor tissues. The tumor cells and vascu-
\end{abstract}

Correspondence to: Professor Bing Hu, Department of Ultrasound in Medicine, Shanghai Institute of Ultrasound in Medicine, Shanghai Jiaotong University Affiliated Sixth People's Hospital, Shanghai Jiaotong University School of Medicine, 600 Yishan Road, Shanghai 200233, P.R. China

E-mail: hub1523@126.com

Key words: ultrasound, low-frequency, contrast agent, cavitation lature were examined by transmission electron microscopy (TEM). Only in the US+UCA group, PI and AUC decreased. The intensity of COX-2 and VEGF in the US+UCA group in immunohistochemical staining and laser confocal microscopy was lower compared to that of the other three groups. More cell apoptosis was found in the US+UCA group compared to the other 3 groups. In the control, UCA and US groups, the tumors had intact vascular endothelium and vessel lumens in TEM. However, lumen occlusion of vessels was observed in the US+UCA group. Twenty-one $\mathrm{kHz}$ low-intensity ultrasound with microbubbles may have anti-angiogenic effects on subcutaneous tumors in nude mice.

\section{Introduction}

Angiogenesis is important in sustaining tumor growth. As a tumor grows, the upregulation of angiogenic factors results in the sprouting of new blood vessels from pre-existing vessels to supply the tumor, but these new vessels fail to mature into a normally functioning vasculature (1). As a consequence, neoangiogenic vessels are fragile, leaky and dysfunctional, and they are targets for various therapeutic modalities (2). Ultrasound has been used for clinical imaging, as well as for its therapeutic actions in physical therapy. It was previously reported that vascular endothelium was destroyed after treatment with ultrasound combined with microbubble contrast agent (UCA) (3). Others found microvessel rupture in vivo in response to acoustic treatment of tissues containing UCAs (4). Continuous 1-MHz low-intensity ultrasound was able to affect the fragile and leaky angiogenic blood vessels in a tumor (5), and 3-MHz ultrasound was more efficacious than $1 \mathrm{MHz}$ for antivascular cancer therapy, in which direct heating of the tumor due to ultrasound absorption is the main mechanism (6). However, in low-frequency ultrasound (7), it is ultrasoundinduced cavitation that may play an important role in the antitumor treatment (8). Collapse cavitation causes capillary destruction and, thus, may be therapeutically beneficial for tumor (9). Cavitation, in a broad sense, refers to ultrasonically induced activity occurring in a liquid or liquid-like material 
that contains bubbles or pockets of gas or vapor. Soft tissue is viscoelastic material, not a pure liquid, and the cavitation thresholds in soft tissue are higher than those in liquid, as endogenous cavitation nuclei are rare in most soft tissues (10). Microbubbles in ultrasound contrast agent (UCA) provide preexisting nuclei and, thus, cavitation threshold of the soft tissue is decreased and microbubbles increase the possibility for ultrasound-induced cavitation and the potential for bioeffects. In vitro studies have been carried out with regard to the effects of low-frequency ultrasound combined with contrast agent (11). However, few researchers have studied these effects in vivo. The objective of the present study was to explore the vascular bioeffects in the tumor tissue of nude mice treated with low-frequency ultrasound combined with a UCA.

\section{Materials and methods}

Animal protocol. The study included three parts, each with 25-35 male nude mice aged 4 weeks and weighing 15-20 g purchased from the Animal Center of the Shanghai Institute of Chinese Academy of Science. All mice were treated and housed according to the approved guidelines (Guidelines for the Care and Use of Laboratory Animals). Following anesthesia by intraperitoneal injection of $0.004 \mathrm{~g}$ ketamine, the mice were secured to a superclean bench according to the principles of aseptic operation. Each mouse was then subcutaneously inoculated with $2 \times 10^{6}$ cells from the DU145 cell line into the flank after local sterilization. The mice continued to be raised at specified pathogen free (SPF) qualification after operation, and were observed at 2-day intervals. Two weeks later, experiments were initiated when the tumors had reached a size of 5-8 mm. Finally, each study included 20 tumor-bearing mice. In the first study, all subcutaneous tumors were examined by contrast-enhanced ultrasonography (CEUS) at the initiation ( 0 week) and completion ( 2 weeks) of the experiments. In the second study, following completion of the experiment, the tumors were excised and examined by immunohistochemistry and confocal laser microscopy to assess the response to low frequency US. In the third study, cell apoptosis of tumor of nude mice was examined by terminal deoxynucleotidyl transferase-mediated dUTP nick end labeling (TUNEL) and tumor cells and micro-vessel were explored by transmission electron microscopy (TEM).

Experimental groupings for tumor therapy and experimental protocol. In each study, 20 tumor-bearing nude mice were randomly divided into four groups, with five mice in each group. The groups were: the A group, negative control (sham treatment); the B group, UCA only; the $\mathrm{C}$ group, low-frequency ultrasound (US); and the D group, US+UCA. A microbubble UCA (SonoVue, Bracco SpA, Milan, Italy) was used. The mice were anesthetized by intraperitoneal injection using $0.3 \mathrm{ml} 1 \%$ pentobarbital sodium. After successful anesthesia, the tumor xenografts were subsequently sonicated using a transducer (Fig. 1) manufactured in the Shanghai Institute of Ultrasound in Medicine at Shanghai Jiaotong University placed on the skin with contact gel (Aquasonic 100; Parker Laboratories Inc., Fairfield, NJ, USA). The diameter of the therapeutic ultrasound transducer was $\sim 13 \mathrm{~mm}$, which could cover the entire tumor (Fig. 2). Low-frequency ultrasound parameters were set at $21 \mathrm{kHz}, 26 \mathrm{~mW} / \mathrm{cm}^{2}$, duty cycle $40 \%$ (on $2 \mathrm{sec}$, off $3 \mathrm{sec}$ ) and duration $3 \mathrm{~min}$ once every other day for two weeks. The intrinsic frequency of the probe is $21 \mathrm{kHz}$. In the pilot study, with the power adjustment to $26 \mathrm{~mW} / \mathrm{cm}^{2}$, no significant heat effect was found on the skin of nude mice, so the cavitation effect on the tumor by low-frequency ultrasound was explored. As in vivo transit time of contrast agents in subcutaneous tumor in CEUS is $\sim 3 \mathrm{~min}$, we set the treatment procedure for $3 \mathrm{~min}$. On the sixteenth day in the US and the US+UCA group, necrosis appeared at the edge of the tumor (arrow) in the control group (Fig. 3), thus, we set the experiment duration to 2 weeks (14 days), to ensure the same experimental conditions with maximum exclusion from other interference factors. The UCA was administered via the tail veins of nude mice by bolus injection. It was composed of a phospholipid shell containing sulfur hexafluoride microbubbles. The dose of contrast agent administered was $0.2 \mathrm{ml}$ per mouse for each treatment. The concentration of contrast agent is $1.8 \times 10^{9} \mathrm{microbubbles} / \mathrm{ml}$.

Contrast-enhanced ultrasonography. CEUS is a useful tool for assessing tumor neovascularity and also for monitoring anti-angiogenic therapies (12). In the first study, at the initiation ( 0 week) and completion ( 2 weeks) of the experiment, the subcutaneous prostate cancer of nude mice was examined by CEUS. CEUS images of the tumors were obtained using Mylab90 instrument (Baisden Medical Co., Italy) by an experienced examiner. The frequency of the probe used was $15 \mathrm{MHz}$. The UCA used was SonoVue, a sulfur hexafluoride UCA (Bracco). The agent $(25 \mathrm{mg}$ ) was shaken for a 1 min with $5 \mathrm{ml}$ of $0.9 \%$ saline solution, and $0.2 \mathrm{ml}$ of this suspension was injected as a bolus manually through a 1-ml syringe placed in the tail vein. Following the bolus injection, the real-time enhancement pattern of contrast agent inside the tumor was observed for 3-5 min and the imaging video was recorded. Images were recorded digitally on an optical disc and analyzed offline. The video was replayed and the area of the whole tumors was chosen as the manually outlined region of interest (ROI) (13). The time-intensity curve (TIC) was drawn automatically with quantitative imaging analysis software (Qontraxt) to obtain the following parameters under intralesion contrast perfusion: time to peak intensity (TTP), peak intensity (PI) and the area under the curve (AUC). Data were processed in the same conditions with the same ultrasound system. The enhancement patterns and TIC results were analyzed by one physician. Qontraxt is an easy-to-use software that reads a time sequence of perfusion images stored in digital format, and allows the objective evaluation of quantitative perfusion parameters. It performs a full map parametric analysis of any portion of an organ during a selected set of frames. For this, the software makes a combination of all the selected frames into a unique set of parametric images. The loop of images is automatically processed after the tissue region and the perfusion period are defined. The resulting parametric maps allow a visual assessment of perfusion properties over the entire selected ROI at once.

Immunohistochemistry. In the second study, the samples of tumors were fixed with formaldehyde, dehydrated with a graded alcohol series and embedded in paraffin. The sections were incubated with primary antibodies against cyclooxygenase-2 


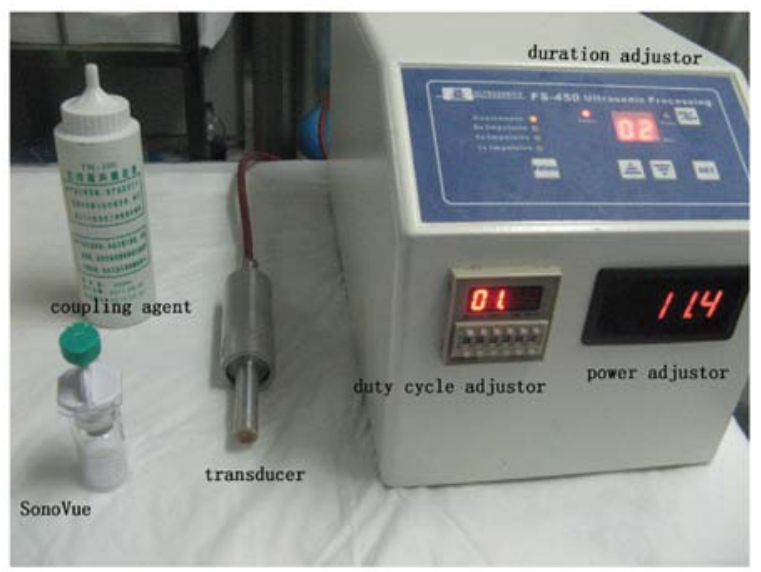

Figure 1. Therapy apparatus for low-frequency ultrasound and experimental setup.

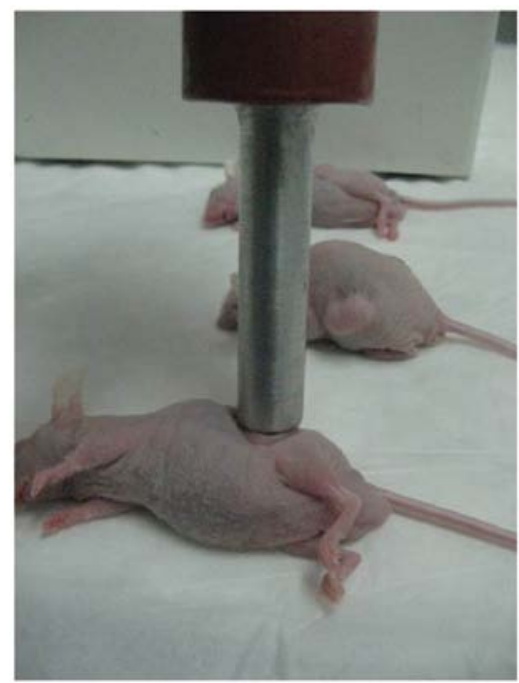

Figure 2. Subcutaneous tumor of nude mice treated by low-frequency ultrasound.

(COX-2) and vascular endothelial growth factor (VEGF; Santa Cruz Biotechnology Inc., Santa Cruz, CA, USA) at a 1:100 dilution, and subsequently incubated with appropriate biotinylated secondary antibody as previously detailed (14). Colorimetric detection was performed using a DAB detection kit (Wuhan Boster Biological Technology Co., Ltd., Wuhan, China). Images were acquired using an Olympus BX51 microscope. The percentage of cells expressing the marker was classified qualitatively, based on the intensity of the immunohistochemical staining and the percent of cells that were stained as follows (15): score 0, low intensity staining in $0-24 \%$ of cells; score 1, low to moderate intensity staining in $25-49 \%$ of cells; score 2, moderate to strong staining in 50-74\% of cells; and score 3 , strong intensity staining in $75-100 \%$ of cells.

Confocal laser microscopy. Microassessment of vascular endothelium can be achieved in selected reasonable sized areas from tissue sections using laser confocal microscopy. Fluorescence expression and distribution pattern were observed using an Olympus FluoView FV500 confocal laser

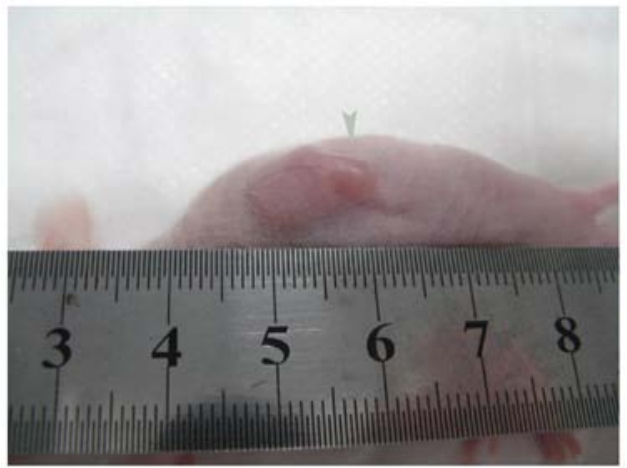

Figure 3. Necrosis at the edge of a tumor (arrow) in the control group.

microscope. The digital image subtraction method was devised to eliminate auto fluorescence. Slices were coded so that analyses could be performed without knowing which treatment each individual animal had received. For each sample, RFP expression and therapy efficiency were evaluated in six randomly chosen fields per section. After thorough washing in normal Tyrode's solution, samples were directly embedded in optimal cutting temperature compound and quickly frozen in liquid nitrogen for VEGF and COX-2 determination using confocal microscopy. Frozen $4 \mu \mathrm{m}$-thick sections were spread over glass slides, allowed to dry for $1 \mathrm{~h}$ and stored desiccated at $-80^{\circ} \mathrm{C}$ until use. Tumor preparations were loaded with either $12.5 \mu \mathrm{M}$ fluo-3 acid or $10 \mu \mathrm{M}$ sodium green acid for $15 \mathrm{~min}$ at room temperature. After several washes in Tyrode's buffer, sections were mounted with a coverslip in $50 \%$ glycerol in phosphate-buffered saline (PBS). Excitation and emission wavelengths were $488 \mathrm{~nm}(10 \%)$ and >530 nm, respectively.

TUNEL staining assay. Apoptosis is an organized process of cell death, which occurs naturally. The induction of apoptosis is of interest in research, as it can be used to evaluate the efficiency of tumor treatment (16). Ultrasound exposure can result in cellular and tissue damage and cell apoptosis $(17,18)$. The third study was performed to examine the potential apoptosis of sonication to tumor-bearing nude mice. Apoptotic cells were detected in deparaffinized tissue sections using a Fluorescein-based In Situ Cell Death Detection Kit (Roche Applied Science, Indianapolis, IN, USA) according to the manufacturer's protocols. Sections were imaged by confocal microscopy for the presence of fluorescein-positive nuclei. The populations were quantified as a percentage of the total cells present in the samples.

Transmission electron microscopy. Also in the third study, each tumor sample $\sim 1 \mathrm{~mm}^{3}$ for transmission electron microscopy (TEM) was fixed in $2 \%$ glutaraldehyde and PBS for $2 \mathrm{~h}$ at $4^{\circ} \mathrm{C}$ followed by PBS buffer and washed twice for $10 \mathrm{~min}$. After treatment with $1 \%$ osmium tetroxide in PBS specimens were fixed in $4^{\circ} \mathrm{C}$ for $2 \mathrm{~h}$ and dehydrated with $30 \%$, followed by $50 \%$, followed by $70 \%$ ethanol three times each for $10 \mathrm{~min}$. The samples were then embedded in propylene oxide for $2 \mathrm{~h}$ and stained with lead citrate E. Finally, after sectioning, specimens were examined using TEM (Philips CM-120; Philips, Eindhoven, The Netherlands). 

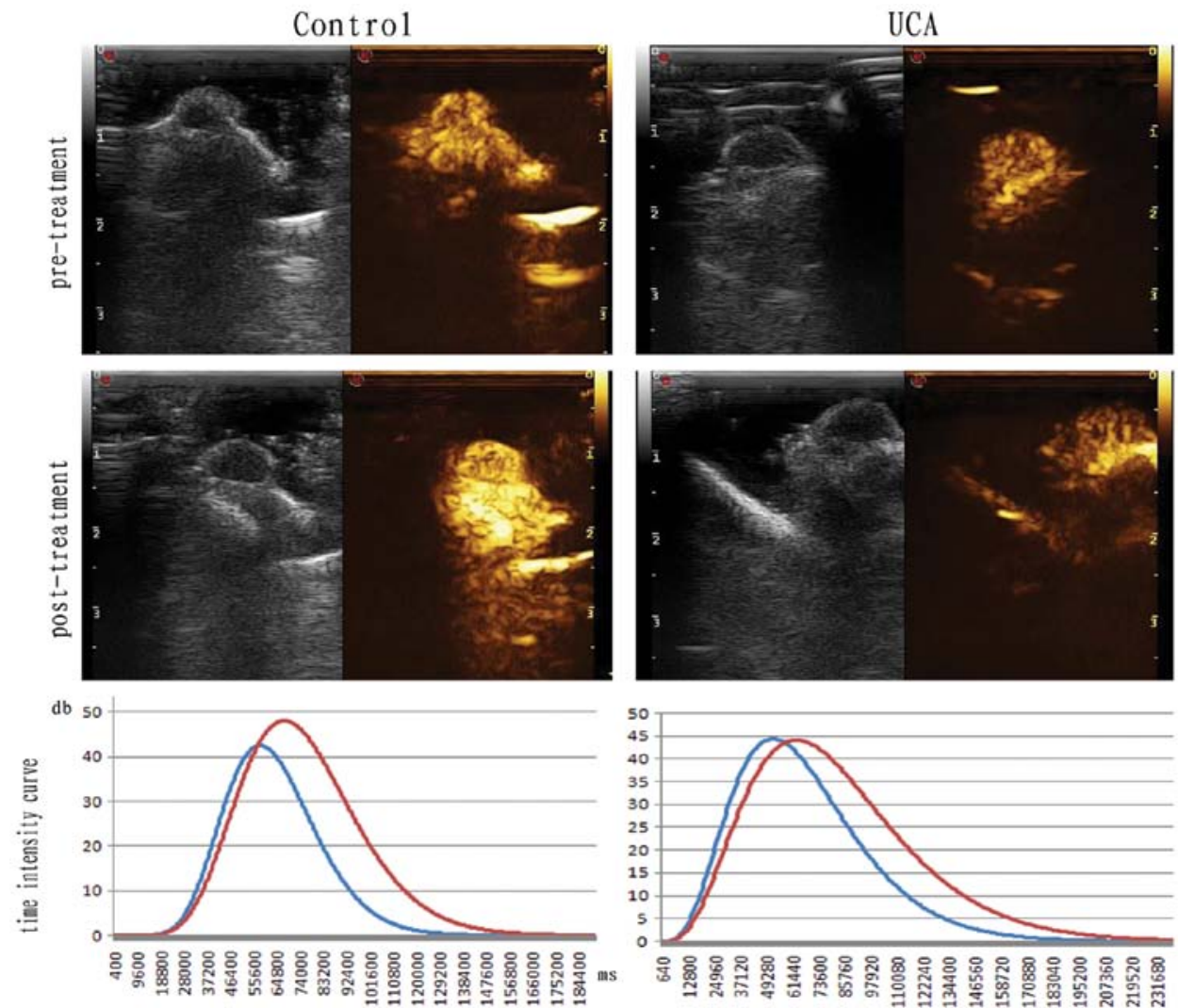

Figure 4. Representative images and curves of tumors in CEUS prior to and following treatment in the control and the UCA group. Blue line, pre-treatment; red line, post-treatment.

US
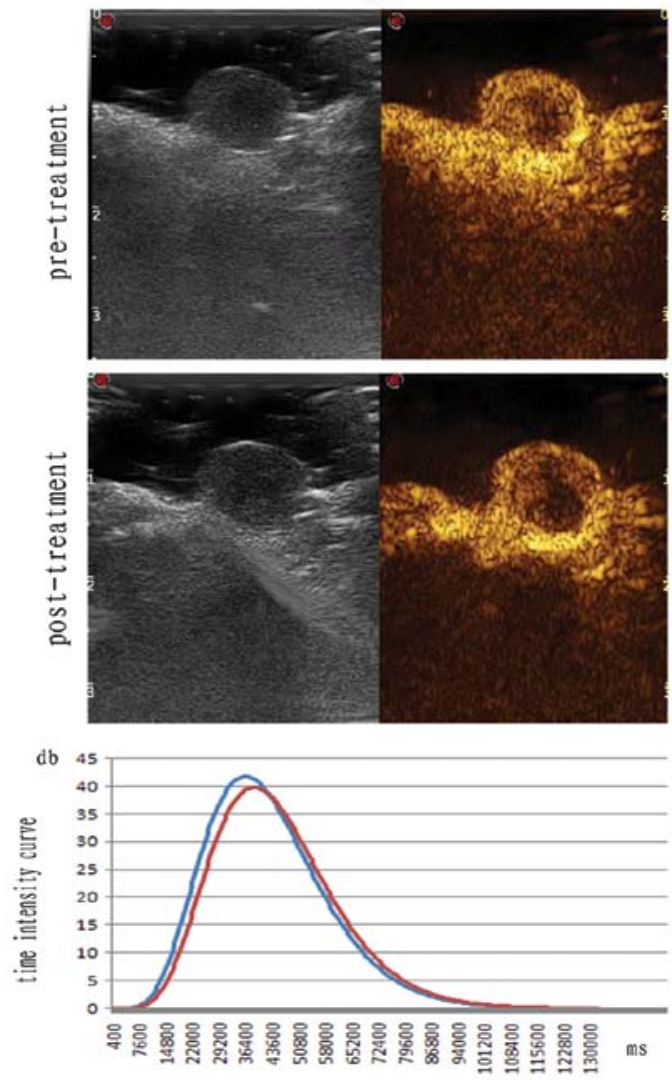

US+UCA
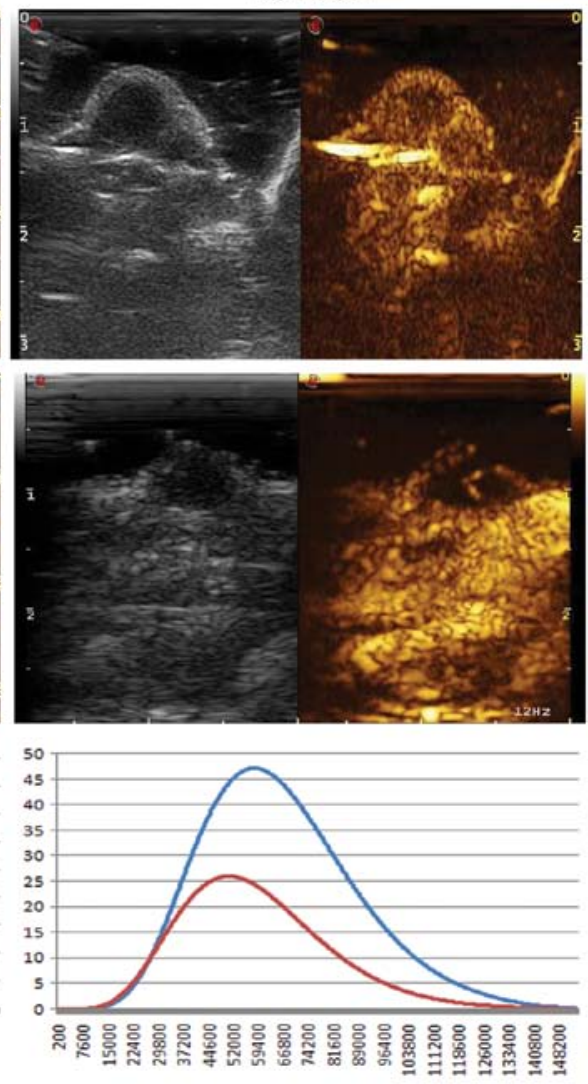

Figure 5. Representative images and curves of tumors in CEUS prior to and following treatment in the US and the US+UCA group. Blue line, pre-treatment; red line, post-treatment. 


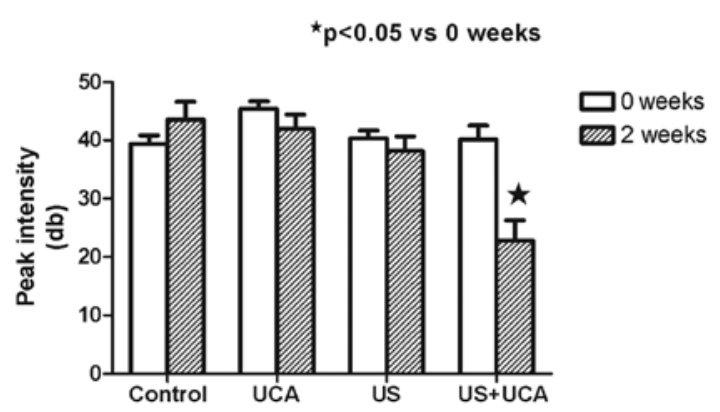

Figure 6. Peak intensity (PI) of the control, UCA, US and US+UCA groups at initiation ( 0 weeks) and completion ( 2 weeks) of the experiments. After 2 weeks of treatment, the PI of the US+UCA group decreased significantly $\left({ }^{\star} \mathrm{t}=4.127, \mathrm{P}<0.05\right)$. In the other 3 groups, there were no significant differences between 0 weeks and 2 weeks $(\mathrm{P}>0.05)$.

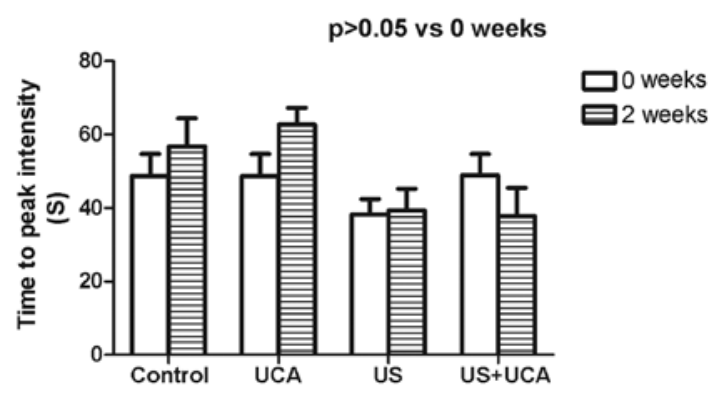

Figure 7. Time to peak intensity in the control, UCA, US and US+UCA groups at 0 weeks and 2 weeks. In the 4 groups, there were no significant differences between 0 weeks and 2 weeks $(\mathrm{P}>0.05)$.

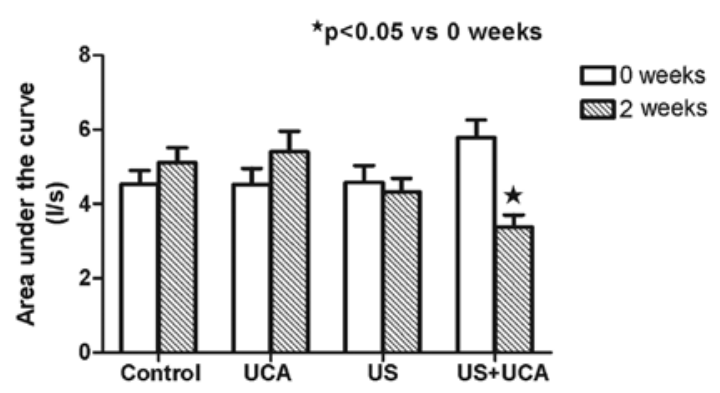

Figure 8. Area under curve (AUC) of the control, UCA, US and US+UCA groups at 0 weeks and 2 weeks. After 2 weeks of treatment, the AUC of the US+UCA group decreased significantly $\left({ }^{\star} t=4.117, \mathrm{P}<0.05\right)$. In the other 3 groups, there were no significant differences between 0 weeks and 2 weeks $(\mathrm{P}>0.05)$.

Statistical analysis. Statistical analysis was performed using SPSS version 11.0 (SPSS Inc., Chicago, IL, USA). The Student's t-test was used to make a statistical comparison between groups. All testing was carried out using Prism 3.0 (GraphPad, San Diego, CA, USA). Error bars were displayed as standard error above the mean. Statistical significance was determined using $\mathrm{P}=0.05$.

\section{Results}

Contrast-enhanced ultrasonography. In the first study, only in the US+UCA group, PI and AUC decreased. The PI of the
Table I. Peak intensity on CEUS prior to and following treatment in each group $(\mathrm{dB})$.

\begin{tabular}{lcccc}
\hline Group & 0 weeks & 2 weeks & t & P-value \\
\hline Control & $39.4 \pm 1.435$ & $43.6 \pm 2.993$ & 1.265 & 0.2414 \\
UCA & $45.4 \pm 1.288$ & $42.1 \pm 2.408$ & 1.245 & 0.2484 \\
US & $40.4 \pm 1.288$ & $38.2 \pm 2.478$ & 0.788 & 0.4536 \\
US+UCA & $40.2 \pm 2.311$ & $22.8 \pm 3.527$ & 4.127 & 0.0033 \\
\hline
\end{tabular}

Table II. Time to peak on CEUS prior to and following treatment in each group (sec).

\begin{tabular}{lllcl}
\hline Group & \multicolumn{1}{c}{0 weeks } & \multicolumn{1}{c}{2 weeks } & \multicolumn{1}{c}{$\mathrm{t}$} & P-value \\
\hline Control & $48.86 \pm 5.87$ & $56.74 \pm 7.67$ & 0.817 & 0.4383 \\
UCA & $48.68 \pm 5.87$ & $62.74 \pm 4.544$ & 1.872 & 0.0985 \\
US & $38.28 \pm 4.13$ & $39.28 \pm 5.97$ & 0.138 & 0.8939 \\
US+UCA & $48.96 \pm 5.729$ & $37.74 \pm 7.739$ & 1.165 & 0.2775 \\
\hline
\end{tabular}

Table III. Area under curve on CEUS prior to and following treatment in each group $(1 / \mathrm{sec})$.

\begin{tabular}{lcrll}
\hline Group & 0 weeks & \multicolumn{1}{c}{2 weeks } & \multicolumn{1}{c}{$\mathrm{t}$} & P-value \\
\hline Control & $4.54 \pm 0.3614$ & $5.12 \pm 0.3992$ & 1.077 & 0.3129 \\
UCA & $4.52 \pm 0.4375$ & $5.2 \pm 0.4087$ & 1.136 & 0.2889 \\
US & $4.58 \pm 0.4532$ & $4.32 \pm 0.3693$ & 0.4447 & 0.6683 \\
US+UCA & $5.78 \pm 0.4831$ & $3.38 \pm 0.3262$ & 4.117 & 0.0034 \\
\hline
\end{tabular}

US+UCA group at the initiation and completion of the experiments was $40.2 \pm 2.311 \mathrm{~dB}$ and $22.8 \pm 3.527 \mathrm{~dB}$ with $\mathrm{t}=4.127$, $\mathrm{P}=0.0033$. The AUC of the US+UCA group at the initiation and completion of the experiments was $5.78 \pm 0.48311 / \mathrm{sec}$ and $3.38 \pm 0.32621 / \mathrm{sec}$ with $\mathrm{t}=4.117, \mathrm{P}=0.0034$. There were no significant changes of PI and AUC in the control, UCA and US groups. There were no significant changes of TTP in the 4 groups (Figs. 4-8; Tables I-III). Insonation of the tumors by US+UCA may be effective in reducing the blood supply of tumors.

Immunohistochemistry. Scores were classified as 0 to 3 , based on the intensity of staining and the percentage of positive cells. The results indicated that the intensity score for the UCA group was the same as that for the control group. This indicated that the UCA alone had no effect on protein expression in the tumor vascular endothelium. In the US group the staining intensity decreased, but there was no significant difference as compared with the control and UCA groups $(\mathrm{P}>0.05)$. However, in the US+UCA group, the staining intensity of COX-2 and VEGF was lower than in the other 3 groups $(\mathrm{P}<0.05)$ (Fig. 9). Therefore, the combination of US and UCA could lead to significant downregulation of the intensity of staining of COX-2 and VEGF protein. 

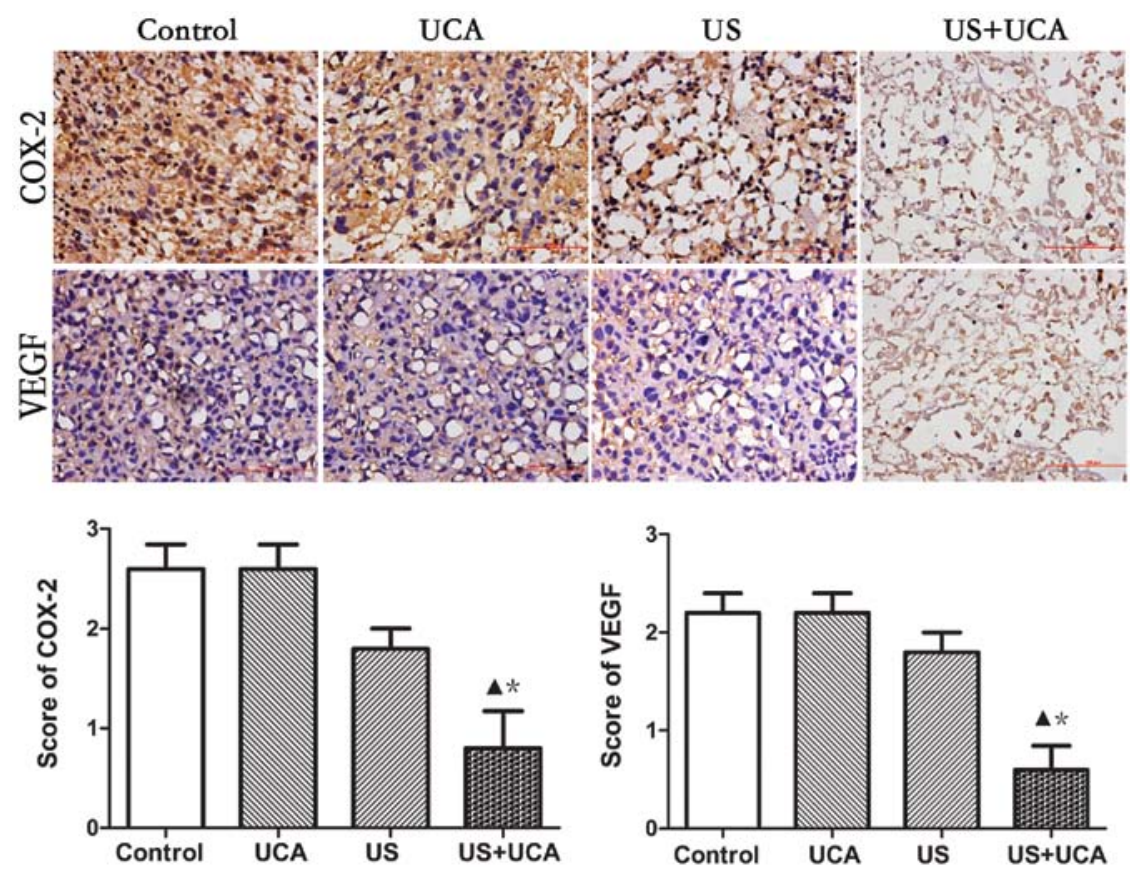

Figure 9. Immunohistochemistry results. In the US+UCA group, the staining intensity of COX-2 and VEGF clearly decreased compared with the other 3 groups $(\mathrm{P}<0.05)$. ${ }^{\wedge} \mathrm{P}<0.05$ vs. control and UCA group. ${ }^{*} \mathrm{P}<0.05$ vs. US group.
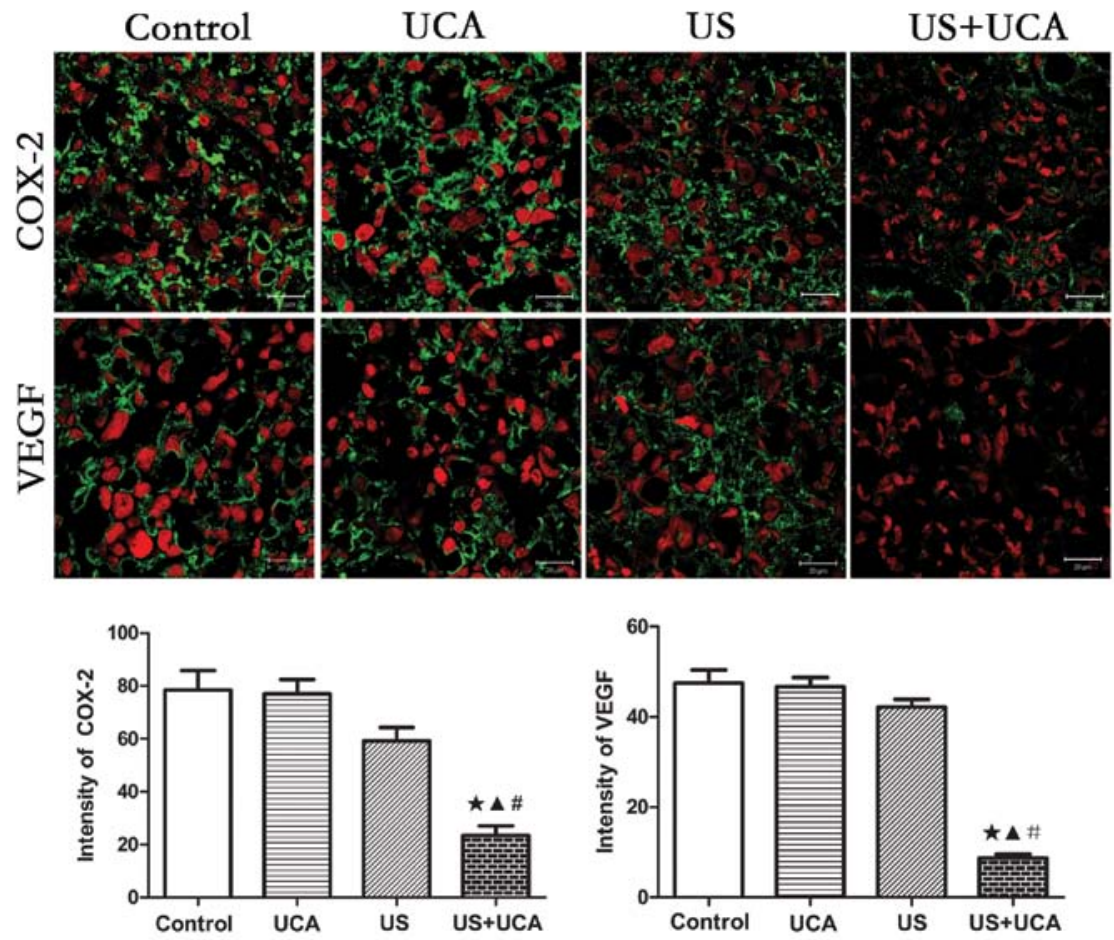

Figure 10. COX-2 and VEGF protein intensity of each group in CLSM. There was a significant difference of COX-2 between the US+UCA and the other 3 groups ( ${ }^{\star} \mathrm{P}<0.001$ vs. control; ${ }^{\wedge} \mathrm{P}<0.001$ vs. UCA; ${ }^{*} \mathrm{P}<0.001$ vs. US). There was a significant difference of VEGF between the US+UCA and the other groups $\left({ }^{\star} \mathrm{P}<0.001\right.$ vs. control; ${ }^{\wedge} \mathrm{P}<0.001$ vs. UCA; ${ }^{~} \mathrm{P}<0.001$ vs. US).

Confocal laser microscopy. In the control group, COX-2 and VEGF protein expression was stronger than in the other 3 groups. The mean intensity values for COX-2 in vascular endothelial cells and cytoplasm in the control, UCA, US and US+UCA groups were 78.44 $\pm 7.367,76.95 \pm 5.518,59.21 \pm 5.113$ and $23.43 \pm 3.608$, respectively. There were significant differences in protein expression among the 4 groups as determined using the ANOVA test, with $\mathrm{F}=21.44$ and $\mathrm{P}<0.0001$. Using the Newman-Keuls multiple comparison test, a significant difference was found between the US+UCA group and other 3 groups $(\mathrm{p}<0.001)$, but there was no significant difference between the control, UCA and US groups $(\mathrm{P}>0.05)$ (Fig. 10). The mean intensity values of VEGF in the vascular endothelial cells and cytoplasm for the control, UCA, US and US+UCA 


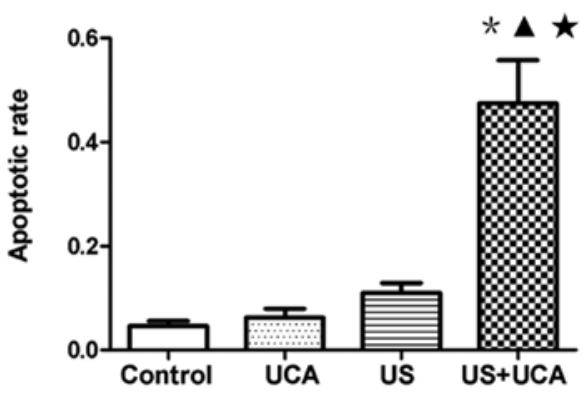

Figure 11. Apoptotic rate in the 4 groups. There were significant differences between the US+UCA and the control, UCA and US groups (*vs. control; $\mathrm{t}=5.108, \mathrm{P}=0.0009 ;{ }^{\star}$ vs. $\mathrm{UCA}, \mathrm{t}=4.849, \mathrm{P}=0.0013 ;{ }^{\star} \mathrm{vs}$. US, $\left.\mathrm{t}=4.267, \mathrm{P}=0.0027\right)$.

groups were $47.51 \pm 2.905,46.66 \pm 2.046,42.17 \pm 1.733$ and $8.71 \pm 0.8691$, respectively. There were significant differences in protein expression among the four groups as determined using the ANOVA test, with $\mathrm{F}=83.67$ and $\mathrm{P}<0.0001$. Using the Newman-Keuls multiple comparison test, a significant difference was found between the US+UCA group and the other 3 groups $(\mathrm{P}<0.001)$, but there was no significant difference between the control, UCA and US groups (P>0.05) (Fig. 10).

TUNEL staining. In the third study, the average apoptotic rates of tumor cells in the control, UCA, US and US+UCA groups were: $4.6 \pm 1,6.2 \pm 1.72,11 \pm 1.9$ and $47.4 \pm 8.3 \%$, respectively. There was a significant difference between the US+UCA and the control, UCA, US groups with $\mathrm{t}=5.108, \mathrm{P}=0.0009$; $\mathrm{t}=4.849$, $\mathrm{P}=0.0013$ and $\mathrm{t}=4.267, \mathrm{P}=0.0027$, respectively (Fig. 11).
Transmission electron microscopy. TEM revealed apparent apoptotic bodies and vascular lumen occlusion in the US+UCA group. Most tumor cells were found normal in the other 3 groups. Intact vascular lumen and normal erythrocytes in the tumor vessels were also found in the control, UCA and US groups (Fig. 12).

\section{Discussion}

A critical event in tumor growth and progression is the upregulation of angiogenesis. Thus, targeting angiogenesis has become an attractive treatment modality in cancer medicine. In the present study, we carried out a novel antitumor study in which subcutaneous tumors implanted in nude mice were exposed to $21 \mathrm{kHz}$ ultrasound, with a pressure amplitude of $26 \mathrm{~mW} / \mathrm{cm}^{2}$, for $3 \mathrm{~min}$ in the presence of the microbubble contrast agent SonoVue. After 2 weeks of treatment, we used CEUS, immunohistochemistry, confocal laser microscopy, TUNEL and TEM, respectively to assess the outcome of the treatment.

Clinical trials have shown that CEUS can be used to assess the anticancer efficacy of antiangiogenic treatments (12). The PI calculated from the TIC is considered to represent flux per unit volume of a scanning lesion. Thus, analyzing the PI using CEUS in tumor parenchyma may aid in the evaluation of the efficacy of anti-angiogenesis treatment of tumors (19). TIC on the CEUS can be used to quantitatively assess tumor microcirculation and reduce the subjectivity of ultrasonography examinations. PI and AUC on the TIC on CEUS can provide non-invasive parameters for evaluation of tumor vascularity
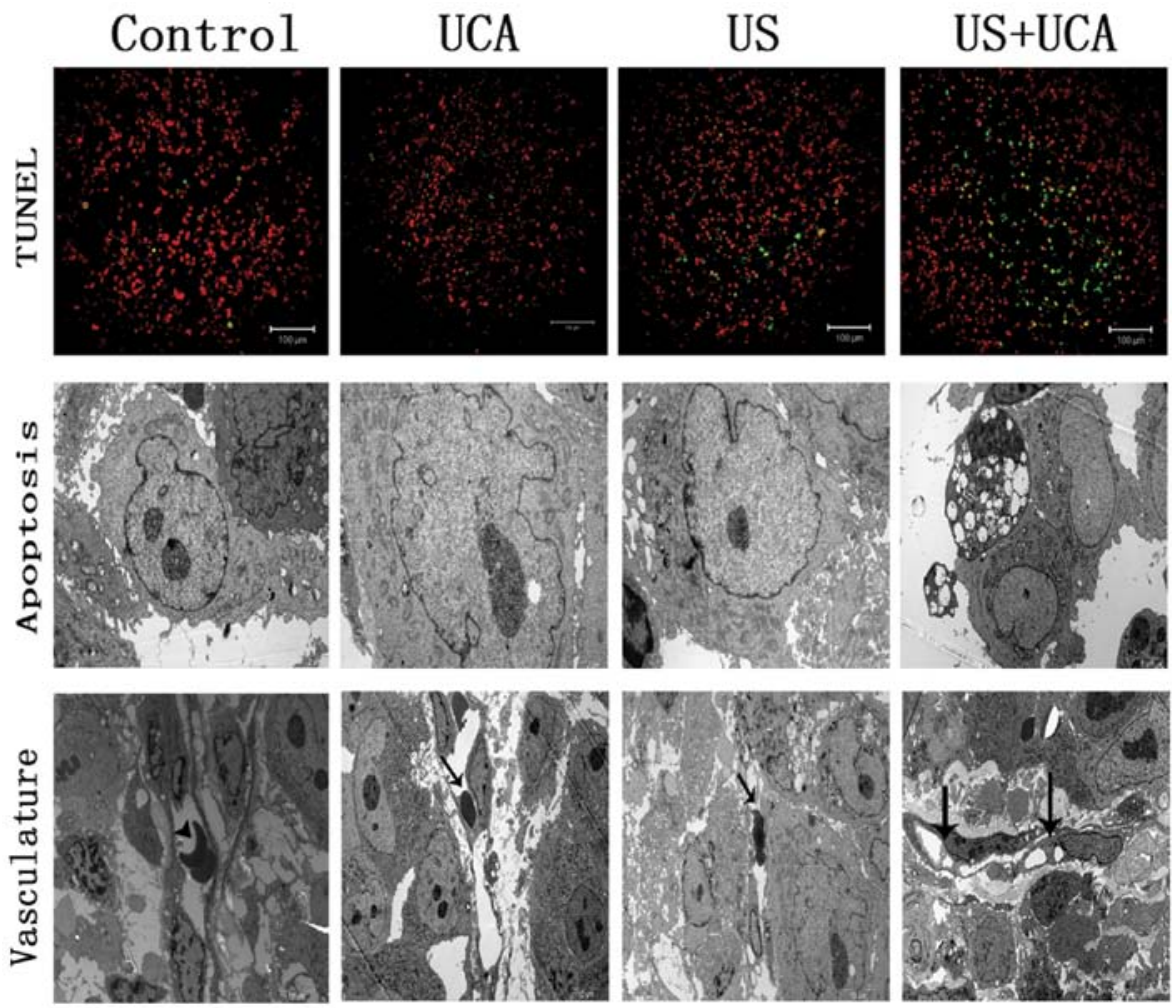

Figure 12. TUNEL staining revealed green staining in the US+UCA group after 2 weeks of treatment. TEM results; tumor cells of the 4 groups by TEM at 2 weeks. Normal tumor cells in the control, UCA and US groups. Increased apoptosis in US+UCA. Microvessels in the control (arrow head), UCA (arrow) and US (arrow) groups had an intact vascular lumen and normal erythrocytes in the vessels; lumen occlusion (arrows) was observed in the US+UCA group. 
as PI and AUC were positively correlated with microvessel density (MVD) (20). In the present study, a decreased PI and AUC in the contrast curve were observed following treatment with low-frequency ultrasound combined with contrast agent. According to a study by Wilhelm et al (21), the antiangiogenic agent can inhibit angiogenesis and reduce MVD. Lavisse et al (22) also reported that the PI was decreased using an anti-angiogenesis treatment. Tumors can induce the growth of new blood vessels to obtain oxygen and nutrition for their growth, and as a consequence the total blood flow in the tumor increases, which is known as angiogenesis. Following treatment, if the tumor has a poor parenchymal vascular network due to decreased angiogenic activity, atrophy of arterioles in the parenchyma can occur, and decreased blood perfusion per unit volume may decrease the onset of tumor enhancement. This may be the reason why PI and AUC were reduced on CEUS after US+UCA treatment of subcutaneous tumors of nude mice.

In the second study, in order to evaluate the results of the treatment, we used immunohistochemistry and confocal laser microscopy to detect angiogenesis-associated gene proteins, such as VEGF and COX-2 of tumor tissue. VEGF is a primary stimulant for tumor angiogenesis, making it a critical target for cancer therapy. COX-2 is associated with carcinogenesis due to stimulation of cell proliferation, inhibition of apoptosis and enhancement of angiogenesis (23). Inhibition of VEGF and COX-2 can be seen as an attractive therapeutic target in the treatment of cancer. Confocal microscopy constitutes a powerful state-of-the-art technique in the investigation of vessel structure and function in normal and pathological conditions, in relation to tumor treatment. In contrast to conventional wide-field fluorescent and light microscopy, images captured using confocal laser scanning microscopes showed much improved clarity with successful elimination of out-of-focus background noise via the pinhole (24). Confocal fluorescent microscopic studies of tumor tissue have been made possible by the intrinsic green autofluorescent properties of tissue. The results of the present study showed that, following intravenous injection of ultrasound contrast agent, there was a clearly decreased VEGF and COX-2 gene expression in the irradiated tumors of nude mice.

In the third study, tumor cells in the US+UCA group had substantially higher apoptosis, compared with the control, UCA and US groups. Low-frequency ultrasound exposure combined with contrast agent induced substantial apoptosis for tumor cells in the tumor-bearing mice. The use of UCA in addition to low-frequency ultrasound is of significance in antitumor therapeutic applications. In previous research, exposure of cells to ultrasonic cavitation was shown to induce apoptosis in addition to the conventionally reported instantaneous cell lysis and necrotic disintegration $(25)$. Other studies $(26,27)$ also reported that the induction of apoptosis by ultrasound exposure has been directly linked to inertial cavitation by its dependence on the presence of a UCA, which provides cavitation nuclei, and by the influence of different dissolved gases. Using TEM in the present study, lumen occlusion were observed in irradiated vessels in the US+UCA group. However, there were no obvious vascular changes in control, UCA and US groups. UCAs, which are artificially augmented population cavitation nuclei, play an important role in the treatment of murine tumors during anti-vasculature therapy. Insonified by low-frequency ultrasound pressure, bubbles become unstable, collapse and fragment in tissue, and this phenomenon is referred to as the bioeffects of acoustic cavitation. Acoustic cavitation involves the concentration of acoustical energy and its conversion into local mechanical perturbation, which can also damage nearby biological cells and structures such as vascular endothelium and vessel lumens. In our study, observed lumen occlusion of vessel leading to the decreased blood supply may be the major reason why PI and AUC were decreased on CEUS and COX-2 and VEGF expression declined in immunohistochemistry and confocal laser microscopy.

Sonicated by ultrasound, bubble expansion significantly distended the vessel to 2.7 times its original diameter (28). The bubble then collapsed at $1.05 \mu$ s leading to almost axially symmetric vessel invagination. The diameter of the vessel at maximum invagination is $\sim 0.4$ times its original diameter. Invagination which generates higher strains on the vessel wall than distention, was commonly observed when bubbles collapsed near the vessel wall, which pulled the vessel inward toward the lumen (28). Sonicated by low frequency ultrasound, the non-linear effect of microbubble cavitation is rather strong, and even low intensity US could cause strong biological effects to cells (29). We hypothesize that cumulative effects of vessel invagination produced by substantial microbubble fragmentation irradiated by $21 \mathrm{kHz}$ US, had a tendency to vascular stenosis, and long-term effects will eventually lead to vascular occlusion. In the future, high-speed photomicrography system may be used to study the relationship between the microbubble sonicated by $21 \mathrm{kHz}$ ultrasound and the blood vessel in vivo.

In the UCA alone group in our study, COX-2 and VEGF expression was the same as that in the control group when assayed using immunohistochemistry and laser confocal microscopy. The reason for this may be that after the UCA microbubbles were injected through the tail vein, they went through the whole body and were excreted through the respiratory tract. The diameter of bubbles was about $2.5 \mu \mathrm{m}$, which was smaller than the red blood cells, but larger than the vascular endothelial gap. Thus, the bubbles seldom penetrated into the tissue spaces. Therefore, in the UCA alone group these bubbles had little effect on vascular endothelium and tumor tissue. The protein expression detected by immunohistochemistry and laser confocal microscopy was similar to the control group.

There were some limitations to our study. First, although US in combination with UCA had an effect on the vessel that was related to protein expression in the tumor tissue, US alone had a few effects on the tumor tissue. Thus, the exact mechanism of vascular damage has not been fully elucidated in our study and requires further research. Second, potential adverse effects on blood vessels in normal tissues were not investigated in the US+UCA group and thus require further exploration in the future.

In general, low-frequency ultrasound in combination with contrast agent was found to be effective in decreasing the PI and AUC of contrast ultrasound imaging and in reducing the expression of VEGF or COX-2 in the vascular endothelium and cytoplasm. More apoptosis was also found in the US+UCA group. Changes to blood vessels may be induced by insonation of $21 \mathrm{kHz}$ ultrasound when they contain exogenous intraluminal UCA. However, the exact mechanisms of low-frequency 
ultrasound and contrast agent on tumor angiogenesis remain to be identified. The interaction of microbubbles with tissue remains a subject of extensive theoretical and experimental study, and is particularly geared towards the optimization of local tumor therapy.

\section{Acknowledgements}

This study was supported in part by the National Natural Science Foundation of China (81271597) and the Key Basic Research Project of Shanghai Science and Technology Commission (10JC1412600). The authors thank Mao Xin and Xie Guo Ming for helping with the tumor cell culture and tumor inoculation. We also thank Zhen Ming Xia and Luan Yan Yan for their help with the contrast-enhanced ultrasonography.

\section{References}

1. Samant RS and Shevde LA: Recent advances in anti-angiogenic therapy of cancer. Oncotarget 2: 122-134, 2011.

2. Sherwood LM, Parris EE and Folkman J: Tumor angiogenesis: therapeutic implications. N Engl J Med 285: 1182-1186, 1971.

3. Hwang JH, Brayman AA, Reidy MA, Matula TJ, Kimmey MB and Crum LA: Vascular effects induced by combined $1-\mathrm{MHz}$ ultrasound and microbubble contrast agent treatments in vivo. Ultrasound Med Biol 31: 553-564, 2005.

4. Skyba DM, Price RJ, Linka AZ, Skalak TC and Kaul S: Direct in vivo visualization of intravascular destruction of microbubbles by ultrasound and its local effects on tissue. Circulation 98: 290-293, 1998.

5. Wood AKW, Ansaloni S, Ziemer LS, Lee WMF, Feldman MD and Sehgal CM: The antivascular action of physiotherapy ultrasound on murine tumors. Ultrasound Med Biol 31: 1403-1410, 2005.

6. Wood AKW, Bunte RM, Price HE, et al: The disruption of murine tumor neovasculature by low-intensity ultrasound-comparison between 1- and 3-MHz sonication frequencies. Acad Radiol 15 : 1133-1141, 2008.

7. Wollina U, Heinig B, Naumann G, Scheibe A, Schmidt WD and Neugebauer R: Effects of low-frequency ultrasound on microcirculation in venous leg ulcers. Indian J Dermatol 56: 174-179, 2011.

8. Barnett SB, Rott HD, ter Haar GR, Ziskin MC and Maeda K: The sensitivity of biological tissue to ultrasound. Ultrasound Med Biol 23: 805-812, 1997.

9. Johnson CA, Sarwate S, Miller RJ and O'Brien WD Jr: A temporal study of ultrasound contrast agent-induced changes in capillary density. J Ultrasound Med 29: 1267-1275, 2010.

10. Yang $X$ and Church CC: A model for the dynamics of gas bubbles in soft tissue. J Acoust Soc Am 118: 3595-3606, 2005.

11. Hutcheson J, Schlicher R, Hicks H and Prausnitz M: Saving cells from ultrasound-induced apoptosis: quantification of cell death and uptake following sonication and effects of targeted calcium chelation. Ultrasound Med Biol 36: 1008-1021, 2010.

12. Lassau N, Chami L, Benatsou B, Peronneau P and Roche A: Dynamic contrast-enhanced ultrasonography (DCE-US) with quantification of tumor perfusion: a new diagnostic tool to evaluate the early effects of antiangiogenic treatment. Eur Radiol 17: F89-F98, 2007.
13. Paprottka P, Cyran C, Zengel P, et al: Non-invasive contrast enhanced ultrasound for quantitative assessment of tumor microcirculation. Contrast mixed mode examination vs. only contrast enhanced ultrasound examination. Clin Hemorheol Microcirc 46: 149-158, 2010.

14. Yu SM and Kim SJ: Endoplasmic reticulum stress (ER-stress) by 2-deoxy-D-glucose (2DG) reduces cyclooxygenase-2 (COX-2) expression and $\mathrm{N}$-glycosylation and induces a loss of COX-2 activity via a Src kinase-dependent pathway in rabbit articular chondrocytes. Exp Mol Med 42: 777-786, 2010.

15. Forsberg F, Dicker AP, Thakur ML, et al: Comparing contrastenhanced ultrasound to immunohistochemical markers of angiogenesis in a human melanoma xenograft model: preliminary results. Ultrasound Med Biol 28: 445-451, 2002.

16. Kotturi H, Li J, Branham-O'Connor M, et al: Tumor cells expressing a fusion protein of MULT1 and Fas are rejected in vivo by apoptosis and NK cell activation. Gene Ther 15: 1302-1310, 2008.

17. Feril LB Jr and Kondo T: Biological effects of low intensity ultrasound: the mechanism involved, and its implications on therapy and on biosafety of ultrasound. J Radiat Res 45: 479-489, 2004.

18. Miller DL and Dou C: Induction of apoptosis in sonoporation and ultrasonic gene transfer. Ultrasound Med Biol 35: 144-154, 2009.

19. Forsberg F, Ro RJ, Fox TB, et al: Contrast enhanced maximum intensity projection ultrasound imaging for assessing angiogenesis in murine glioma and breast tumor models: a comparative study. Ultrasonics 51: 382-389, 2011.

20. Wang J, Lv F, Fei X, et al: Study on the characteristics of contrastenhanced ultrasound and its utility in assessing the microvessel density in ovarian tumors or tumor-like lesions. Int J Biol Sci 7: 600-606, 2011.

21. Wilhelm SM, Carter C, Tang LY, et al: BAY 43-9006 exhibits broad spectrum oral antitumor activity and targets the RAF/ MEK/ERK pathway and receptor tyrosine kinases involved in tumor progression and angiogenesis. Cancer Res 64: 7099-7109, 2004.

22. Lavisse S, Lejeune P, Rouffiac V, et al: Early quantitative evaluation of a tumor vasculature disruptive agent AVE8062 using dynamic contrast-enhanced ultrasonography. Invest Radiol 43: 100-111, 2008.

23. Chan TA: Nonsteroidal anti-inflammatory drugs, apoptosis, and colon-cancer chemoprevention. Lancet Oncol 3: 166-174, 2002.

24. Lu J, Min W, Conchello JA, Xie XS and Lichtman JW: Superresolution laser scanning microscopy through spatiotemporal modulation. Nano Lett 9: 3883-3889, 2009.

25. Ashush H, Rozenszajn LA, Blass M, et al: Apoptosis induction of human myeloid leukemic cells by ultrasound exposure. Cancer Res 60: 1014-1020, 2000.

26. Honda H, Zhao QL and Kondo T: Effects of dissolved gases and an echo contrast agent on apoptosis induced by ultrasound and its mechanism via the mitochondria-caspase pathway. Ultrasound Med Biol 28: 673-682, 2002.

27. Feril LB, Kondo T, Zhao QL, et al: Enhancement of ultrasoundinduced apoptosis and cell lysis by echo-contrast agents. Ultrasound Med Biol 29: 331-337, 2003.

28. Chen H, Brayman AA, Bailey MR and Matula TJ: Blood vessel rupture by cavitation. Urol Res 38: 321-326, 2010.

29. Tian ZM, Wan MX, Lu MZ, Wang XD and Wang L: The alteration of protein profile of Walker 256 carinosarcoma cells during the apoptotic process induced by ultrasound. Ultrasound Med Biol 31: 121-128, 2005. 